\title{
Experimental review of jet physics in hadronic collisions
}

\author{
Mario Martínez ${ }^{\mathrm{a}}$ \\ ICREA/IFAE, Institut de Física d'Altes Energies, Barcelona, Spain
}

Received: 11 September 2008 / Revised: 2 December 2008 / Published online: 9 January 2009

(C) Springer-Verlag / Società Italiana di Fisica 2009

\begin{abstract}
In this contribution, a comprehensive review of the main aspects of high $p_{\mathrm{T}}$ jet physics in Run II at the Tevatron is presented. Recent measurements on inclusive jet production are discussed using different jet algorithms and covering a wide region of jet transverse momentum and jet rapidity. Several measurements, sensitive to a proper description of soft gluon radiation and the underlying event in hadron collisions, are shown. Finally, high $p_{\mathrm{T}}$ prompt photon measurements, and studies on the production of electroweak bosons in association with jets in the final state are discussed.
\end{abstract}

PACS 12.38.Aw $\cdot 13.85 .-\mathrm{t} \cdot 13.87 .-\mathrm{a}$

\section{Inclusive jet production}

The measurement of the inclusive jet cross section in $p \bar{p}$ collisions at $\sqrt{s}=1.96 \mathrm{TeV}$ constitutes a stringent test of perturbative QCD (pQCD) predictions over almost nine orders of magnitude. The increased center-of-mass energy and integrated luminosity in Run II at the Tevatron, compared to Run I, allows to extend the measured jet cross section to jets with transverse momentum $p_{\mathrm{T}}^{\text {jet }}$ above $650 \mathrm{GeV} / c$, and to search for signals of quark compositeness down to $\sim 10^{-19} \mathrm{~m}$.

The pQCD calculations are written as matrix elements, describing the hard interaction between partons, convoluted with parton density functions (PDFs) in the proton and antiproton that require input from the experiments. Inclusive jet cross section measurements from Run I [1, 2], performed in different jet rapidity regions, have been used to partially constrain the gluon distribution in the proton. The pQCD predictions are affected by the still limited knowledge on the gluon PDF, which translates into a large uncertainty on the theoretical cross sections at high $p_{\mathrm{T}}^{\text {jet }}$. A proper comparison with the $\mathrm{pQCD}$ predictions at the parton level also requires

a e-mail: mmp@ifae.es an adequate modeling of soft-gluon contributions which become important at low $p_{\mathrm{T}}^{\text {jet }}$.

In Run II, both CDF and D0 experiments have explored new jet algorithms following the theoretical work indicating that the cone-based jet algorithm employed in Run I is not infrared safe and compromises a future meaningful comparison with pQCD calculations at NNLO. The CDF experiment has published results [3-6] on inclusive jet production using both the $k_{\mathrm{T}}[7,8]$ and the midpoint $[9,10]$ algorithms. While the $k_{\mathrm{T}}$ algorithm is infrared safe to all orders in perturbation theory, the midpoint algorithm still suffers from some infrared sensitivity at higher orders. The measurements are performed for jets with $p_{\mathrm{T}}^{\text {jet }}>54 \mathrm{GeV} / c$ and rapidity in the region $\left|y^{\text {jet }}\right|<2.1$, using $1.0 \mathrm{fb}^{-1}$ of data. Figure 1.1 shows

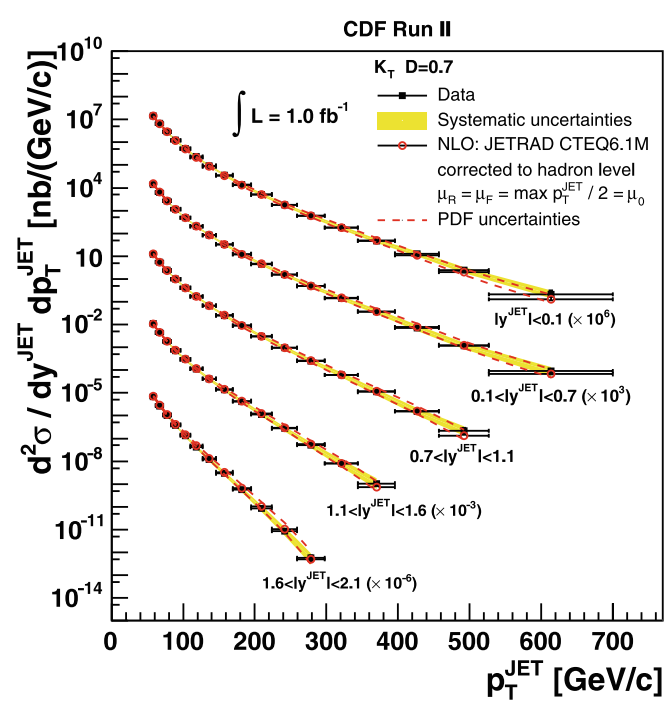

Fig. 1.1 Measured inclusive differential jet cross sections, using the $k_{\mathrm{T}}$ algorithm with $D=0.7$ (black dots) as a function of $p_{\mathrm{T}}^{\text {jet }}$ for jets with $p_{\mathrm{T}}^{\text {jet }}>54 \mathrm{GeV} / c$ in different $\left|y^{\text {jet }}\right|$ regions compared to NLO pQCD predictions (open circles). The shaded bands show the total systematic uncertainty on the measurements. The dashed lines indicate the PDF uncertainty on the theoretical predictions 


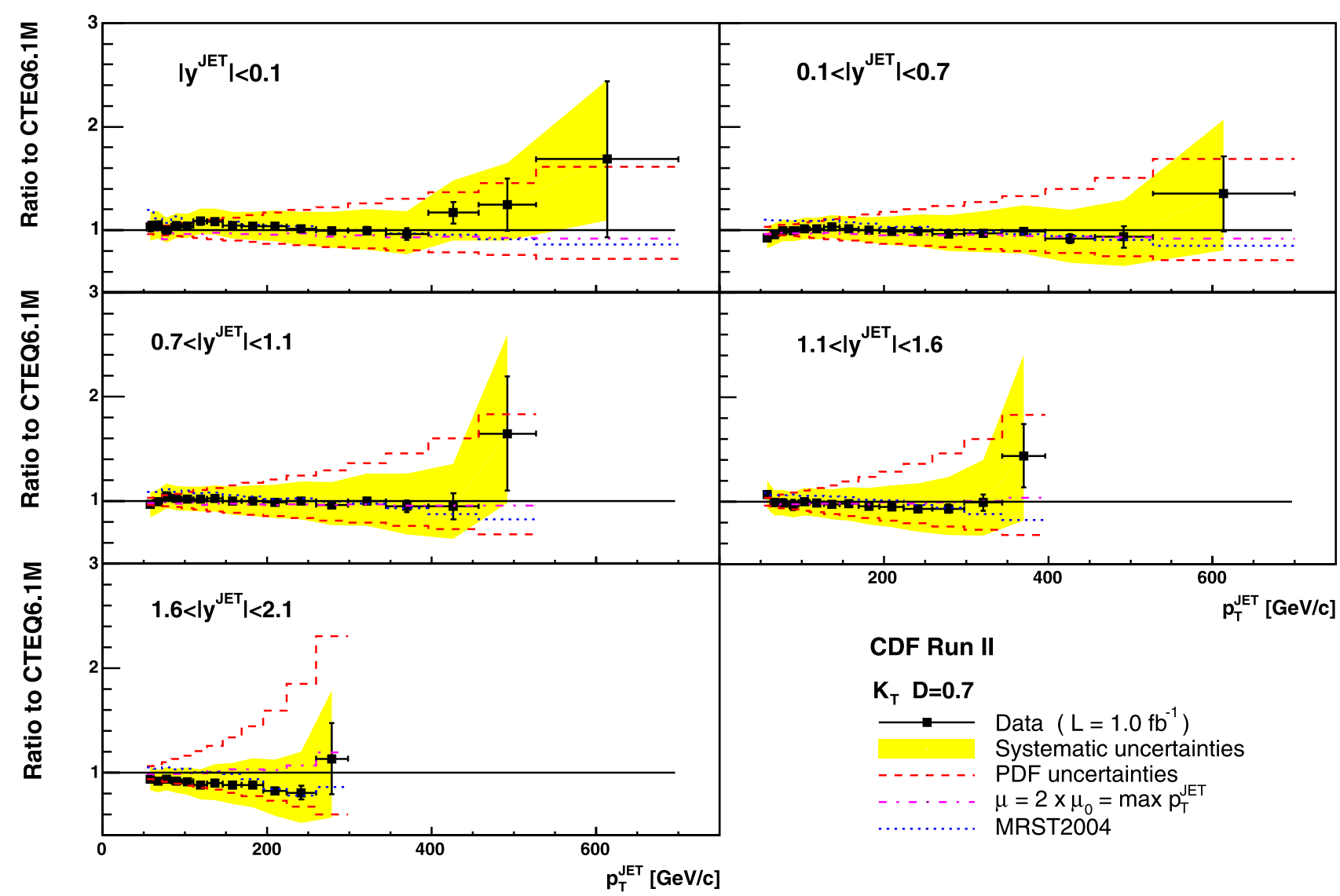

Fig. 1.2 Ratio Data/Theory as a function of $p_{\mathrm{T}}^{\text {jet }}$ in different $\left|y^{\text {jet }}\right|$ regions. The error bars (shaded bands) show the total statistical (systematic) uncertainty on the data. A $6 \%$ uncertainty on the luminosity is not included. The dashed lines indicate the PDF uncertainty on the the-

the measured cross sections using the $k_{\mathrm{T}}$ algorithm,

$k_{\mathrm{T}, i}=p_{\mathrm{T}, i}^{2} ;$

$k_{\mathrm{T},(i, j)}=\min \left(p_{\mathrm{T}, i}^{2}, p_{\mathrm{T}, j}^{2}\right) \cdot \Delta R_{i, j}^{2} / D^{2}$,

where $p_{\mathrm{T}, i}$ denotes the transverse momentum of each particle in the final state, $\Delta R_{i, j}$ is the distances, in $\eta-\phi$ space, between each pair of particles, and $D=0.7$. The cross sections are measured as a function of $p_{\mathrm{T}}^{\mathrm{jet}}$ in five different $\left|y^{\text {jet }}\right|$ regions, compared to NLO pQCD predictions [11]. The measured cross sections decrease by more than seven to eight orders of magnitude as $p_{\mathrm{T}}^{\text {jet }}$ increases. Figure 1.2 shows the ratios data/theory as a function of $p_{\mathrm{T}}^{\text {jet }}$. Good agreement is observed in the whole range in $p_{\mathrm{T}}^{\mathrm{jet}}$ and $y^{\text {jet }}$ between the measured cross sections and the theoretical predictions. ${ }^{1}$ In particular, no significant deviation from the

${ }^{1}$ Previous measurements using the $k_{\mathrm{T}}$ algorithm at the Tevatron [12] observed a marginal agreement with NLO pQCD at low $p_{\mathrm{T}}^{\text {jet }}$ but this discrepancy is removed after non-perturbative corrections are included. oretical predictions. The dotted lines present the ratios of MRST2004 and CTEQ6.1M predictions. The dotted-dashed lines show the ratios of predictions with different renormalization/factorization scales

pQCD prediction is observed for central jets at high $p_{\mathrm{T}}^{\mathrm{jet}}$. In the most forward region, the uncertainty on the measured cross section at high $p_{\mathrm{T}}^{\text {jet }}$, compared to that on the theoretical prediction, indicates that the data will contribute to a better understanding of the gluon PDF. Finally, in the region $0.1<\left|y^{\text {jet }}\right|<0.7$, the analysis is repeated using different values for $D$ in the $k_{\mathrm{T}}$ algorithm: $D=0.5$ and $D=1.0$ (see Fig. 1.3). In both cases, good agreement is observed between the measured cross sections and the NLO pQCD predictions in the whole range in $p_{\mathrm{T}}^{\text {jet }}$. As $D$ decreases, the measurement is less sensitive to contributions from multiple proton-antiproton interactions, and the presence and proper modeling of the underlying event. These measurements show a proper understanding of non-perturbative contributions, as extracted from Monte Carlo models, and support the validity of the $k_{\mathrm{T}}$ algorithm to search for jets in hadron collisions that will be further explored at the LHC energies. 


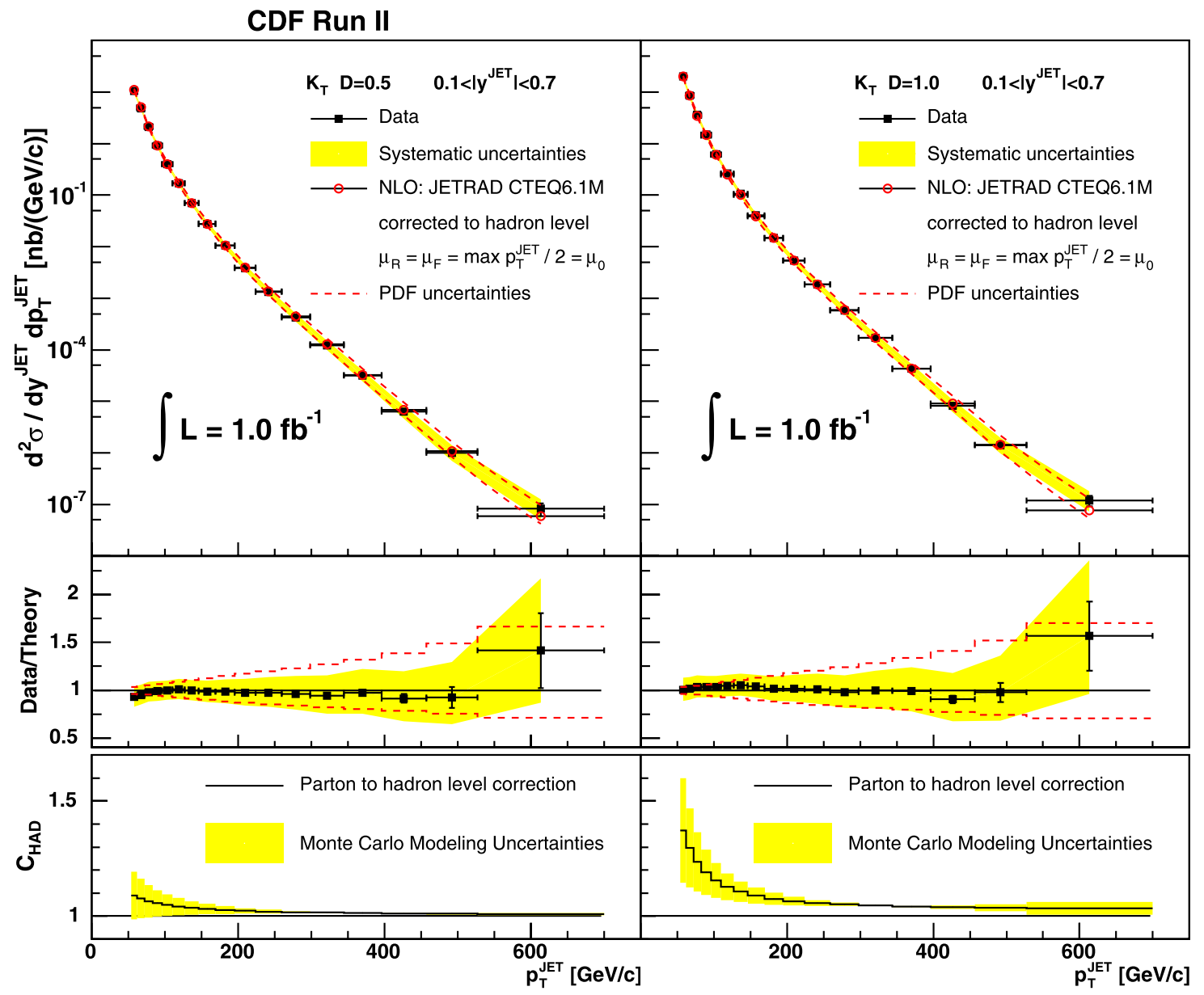

Fig. 1.3 Measured inclusive differential jet cross sections (black dots) as a function of $p_{\mathrm{T}}^{\text {jet }}$ using $D=0.5($ left $)$ and $D=1.0($ right $)$, compared to NLO pQCD predictions. The shaded bands show the total systematic uncertainty on the measurements. The dashed lines indicate the

Similarly, Fig. 1.4 shows the measured inclusive jet cross sections by D0 [13] based on $0.7 \mathrm{fb}^{-1}$ of Run II data. The midpoint jet algorithm has been used with a cone size $R=$ 0.7. The measurements are carried out for jets with $p_{\mathrm{T}}^{\text {jet }}>$ $50 \mathrm{GeV} / c$ in six different rapidity regions up to $\left|y^{\text {jet }}\right|<2.4$. The data are compared to NLO pQCD predictions, as implemented in the NLO++ $[14,15]$ program, with CTEQ6.5M and MRST2004 PDFs sets, and using $p_{\mathrm{T}}^{\mathrm{jet}}$ as the nominal renormalization/factorization scale. Figure 1.5 presents the ratio data vs NLO pQCD predictions as a function of $p_{\mathrm{T}}^{\text {jet }}$ in the different $\left|y^{\text {jet }}\right|$ regions. The measurements are in good agreement with the theoretical predictions within the current PDFs uncertainties. However, the figure also suggests that the data prefer the lower edge of the CTEQ uncertainty band while the measurements are in good agreement with the nominal predictions when MRST2004 PDFs are used.
PDF uncertainty on the theoretical predictions. (bottom) Magnitude of the parton-to-hadron corrections, $C_{\mathrm{HAD}}\left(p_{\mathrm{T}}^{\mathrm{jet}}\right)$, used to correct the NLO pQCD predictions for $D=0.5$ (left) and $D=1.0$ (right). The shaded bands indicate the quoted Monte Carlo modeling uncertainty

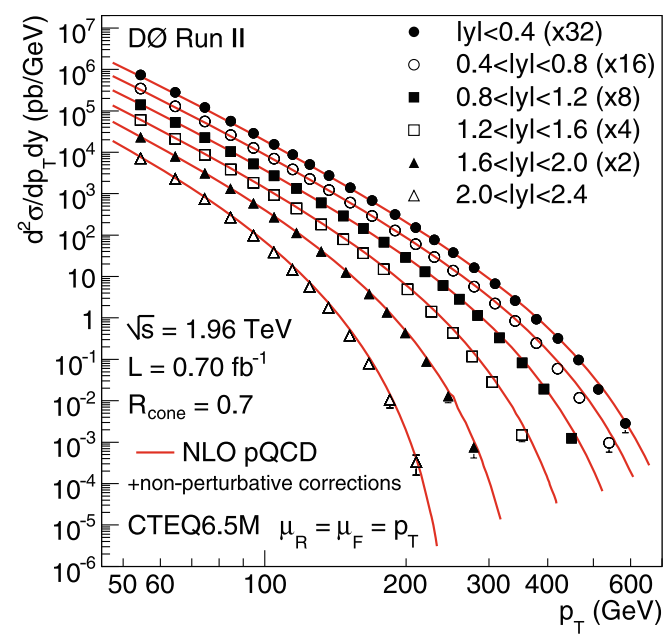

Fig. 1.4 Measured inclusive jet cross section as a function of $p_{\mathrm{T}}^{\text {jet }}$ in different $\left|y^{\text {jet }}\right|$ ranges (dots), compared to pQCD NLO predictions (full lines). For presentation, the measurements are scaled by a given factor 
Fig. 1.5 Ratio data/theory as a function of $p_{\mathrm{T}}^{\text {jet }}$ in different $\left|y^{\text {jet }}\right|$ ranges. The bands indicate the uncertainty on the data and the dashed lines the uncertainty on the NLO prediction using CTEQ6.5M PDFs. The dotted lines show the ratio to MRST2004 PDFs

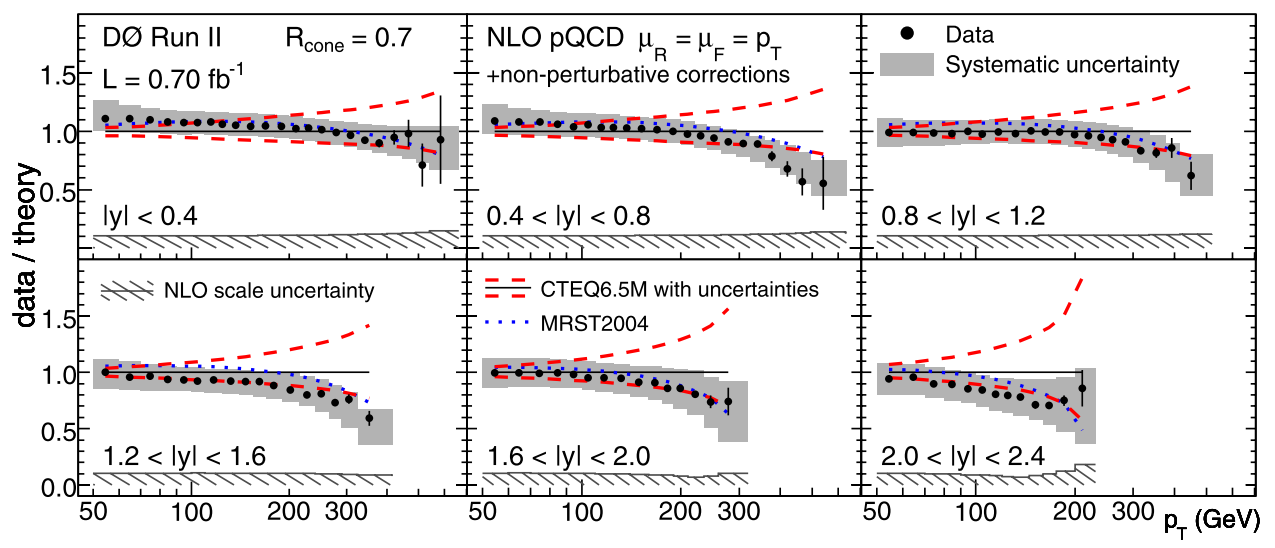

\section{Studies of the underlying event}

As mentioned in the previous section, the hadronic final states at the Tevatron are characterized by the presence of soft underlying emissions, usually denoted as underlying event, in addition to highly energetic jets coming from the hard interaction. The underlying event contains contributions from initial- and final-state soft gluon radiation, secondary semi-hard partonic interactions and interactions between the proton and anti-proton remnants that cannot be described by perturbation theory. These processes must be approximately modeled using Monte Carlo programs tuned to describe the data. The jet energies measured in the detector contain an underlying event contribution that has to be subtracted in order to compare the measurements to $\mathrm{PQCD}$ predictions. Hence, a proper understanding of this underlying event contribution is crucial to reach the desired precision in the measured jet cross sections. In the analysis presented here, the underlying event in dijet events has been studied by looking at regions well separated from the leading jets (see Fig. 2.1), where the underlying event contribution is expected to dominate the observed hadronic activity. Jets have been reconstructed using tracks with $p_{\mathrm{T}}^{\text {track }}>0.5 \mathrm{GeV}$ and $\left|\eta^{\text {track }}\right|<1$ and a cone algorithm with $R=0.7$. Figure 2.2 shows the average track density in the transverse region as a function of $E_{\mathrm{T}}^{\mathrm{jet}}$ of the leading jet for the dijet inclusive sample and for events where the leading jets are forced to be back-to-back in $\phi$, in order to further reduce extra hardgluon radiation. The observed plateau indicates that the underlying event activity is, to first approximation, independent from the hard interaction. The measurements have been compared to the predictions from PYTHIA [16] and HERWIG [17] Monte Carlo programs, including leading-order QCD matrix elements plus initial and final parton showers. The PYTHIA samples have been created using a special set of parameters, denoted as PYTHIA-TUNE A [18], ${ }^{2}$ which in-

${ }^{2}$ PYTHIA-TUNE A Monte Carlo samples are generated using the following tuned parameters in PYTH IA: PARP $(67)=4.0, \operatorname{MSTP}(82)=$

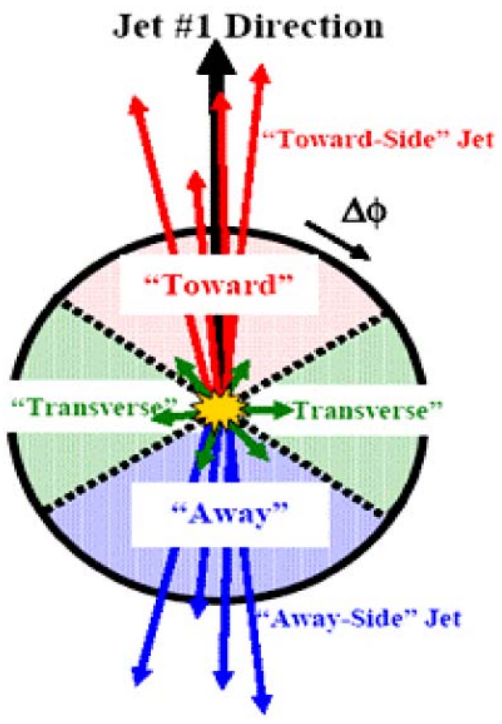

Fig. 2.1 Scheme of the different $\phi$ regions defined around the leading jet

cludes an enhanced contribution from initial-state soft gluon radiation and a tuned set of parameter to control secondary parton interactions. It was determined as a result of similar studies of the underlying event performed using CDF Run I data [18]. PYTHIA-TUNE A describes the hadronic activity in transverse region while HERWIG underestimates the radiation at low $E_{\mathrm{T}}^{\mathrm{jet}}$. Recent results, only available at the time of preparing this proceeding [19], indicate that PYTHIATUNE A is also able to describe the underlying event activity in Drell-Yan events, with a significantly different color configuration in the final state.

4, $\operatorname{PARP}(82)=2.0, \operatorname{PARP}(84)=0.4, \operatorname{PARP}(85)=0.9, \operatorname{PARP}(86)=$ $0.95, \operatorname{PARP}(89)=1800.0, \operatorname{PAR} \mathrm{P}(90)=0.25$. 


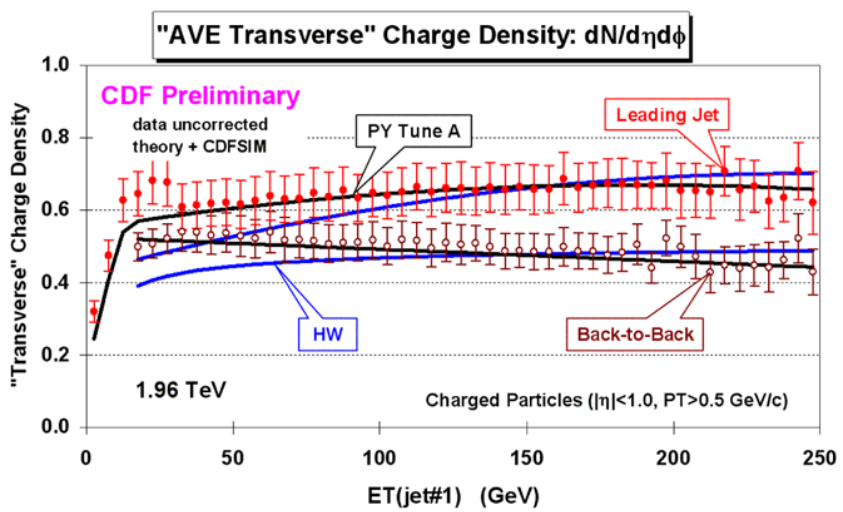

Fig. 2.2 Measured average track density in the transverse region as a function of the $E_{\mathrm{T}}^{\mathrm{jet}}$ of the leading jet. The measurements are compared to different Monte Carlo models

\section{Jet shapes}

The internal structure of jets is dominated by multi-gluon emissions from the primary final-state parton. It is sensitive to the relative quark- and gluon-jet fraction and receives contributions from soft-gluon initial-state radiation and beam remnant-remnant interactions. The study of jet shapes at the Tevatron provides a stringent test of QCD predictions and tests the validity of the models for parton cascades and soft-gluon emissions in hadron-hadron collisions. The CDF experiment has published results [20] on jet shapes for central jets with transverse momentum in the region $37<p_{\mathrm{T}}^{\text {jet }}<380 \mathrm{GeV}$, where jets are searched for using the midpoint ${ }^{3}$ algorithm and a cone size $R=0.7$. The integrated jet shape, $\Psi(r)$, is defined as the average fraction of the jet transverse momentum that lies inside a cone of radius $r$ concentric to the jet cone:

$\Psi(r)=\frac{1}{N} \sum_{\text {jet }} \sum_{\text {jets }} \frac{P_{\mathrm{T}}(0, r)}{P_{\mathrm{T}}(0, R)}, \quad 0 \leq r \leq R$

where $N_{\text {jet }}$ denotes the number of jets, see Fig. 3.1. The measured jet shapes have been compared to the predictions from PYTHIA-TUNE A and HERWIG Monte Carlo programs. In addition, two different PYTHIA samples have been used with default parameters and with and without the contribution from multiple parton interactions (MPI) between proton and antiproton remnants, the latter denoted as PYTHIA-(no MPI), to illustrate the importance of a proper modeling of softgluon radiation in describing the measured jet shapes. Figure 3.2 presents the measured integrated jet shapes, $\Psi(r / R)$, for jets with $37<p_{\mathrm{T}}^{\text {jet }}<45 \mathrm{GeV}$, compared to HERWIG, PYTHIA-TUNE A, PYTHIA and PYTHIA-(no MPI) predictions. Figure 3.3 shows, for a fixed radius $r_{0}=0.3$, the average fraction of the jet transverse momentum outside $r=r_{0}$,

${ }^{3}$ A $75 \%$ merging fraction has been used.

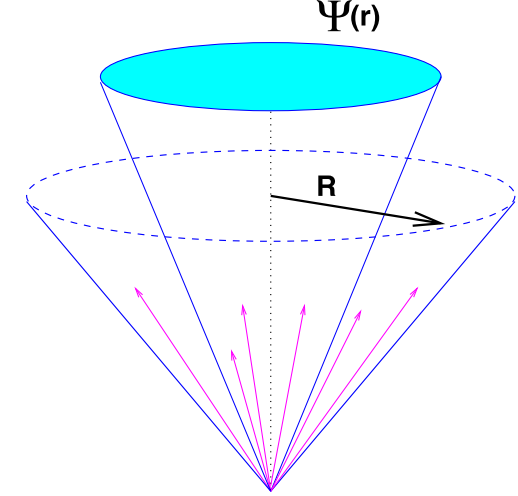

Fig. 3.1 Integrated jet shapes definition

CDF Run II Preliminary

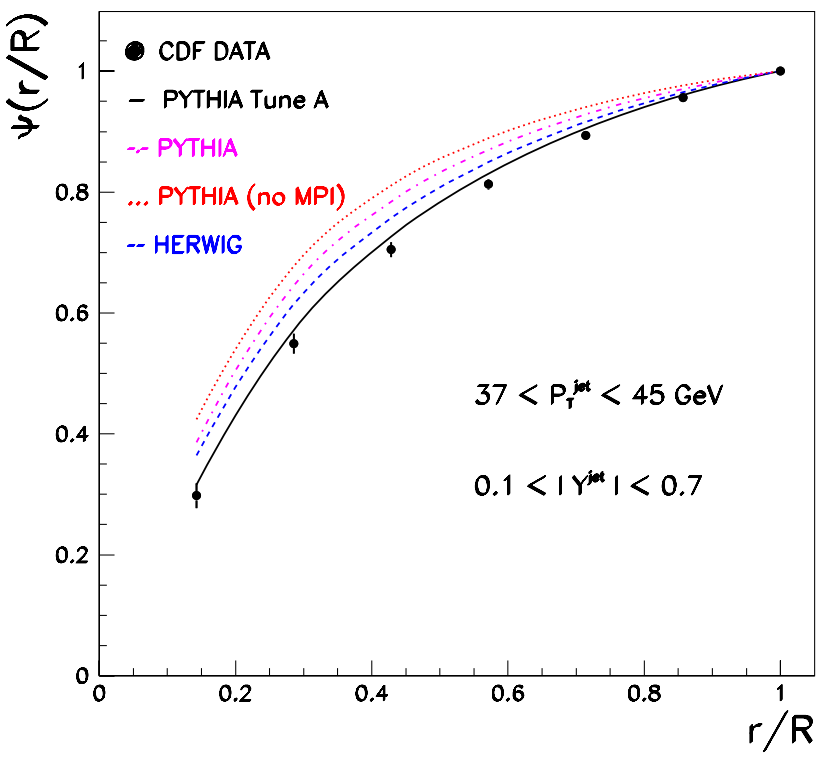

Fig. 3.2 The measured integrated jet shape, $\Psi(r / R)$, in inclusive jet production for jets with $0.1<\left|y^{\text {jet }}\right|<0.7$ and $37 \mathrm{GeV} / c<p_{\mathrm{T}}^{\text {jet }}<45 \mathrm{GeV} / c$. The predictions of PYTHIA-TUNE A (solid lines), PYTHIA (dashed-dotted lines), PYTHIA-(no MPI) (dotted lines) and HERWIG (dashed lines) are shown for comparison

$1-\Psi\left(r_{0} / R\right)$, as a function of $p_{\mathrm{T}}^{\text {jet }}$, where the points are located at the weighted mean in each $p_{\mathrm{T}}^{\text {jet }}$ range. The measurements show that the fraction of jet transverse momentum at a given fixed $r_{0} / R$ increases $\left(1-\Psi\left(r_{0} / R\right)\right.$ decreases $)$ with $p_{\mathrm{T}}^{\text {jet }}$, indicating that the jets become narrower as $p_{\mathrm{T}}^{\text {jet }}$ increases. PYTHIA with default parameters produces jets systematically narrower than the data in the whole region in $p_{\mathrm{T}}^{\text {jet }}$. The contribution from secondary parton interactions between remnants to the predicted jet shapes (shown by the difference between PYTHIA and PYTHIA-(no MPI) predictions) is relatively small and decreases as $p_{\mathrm{T}}^{\text {jet }}$ increases. PYTHIA-TUNE A predictions describe all of the data well. 


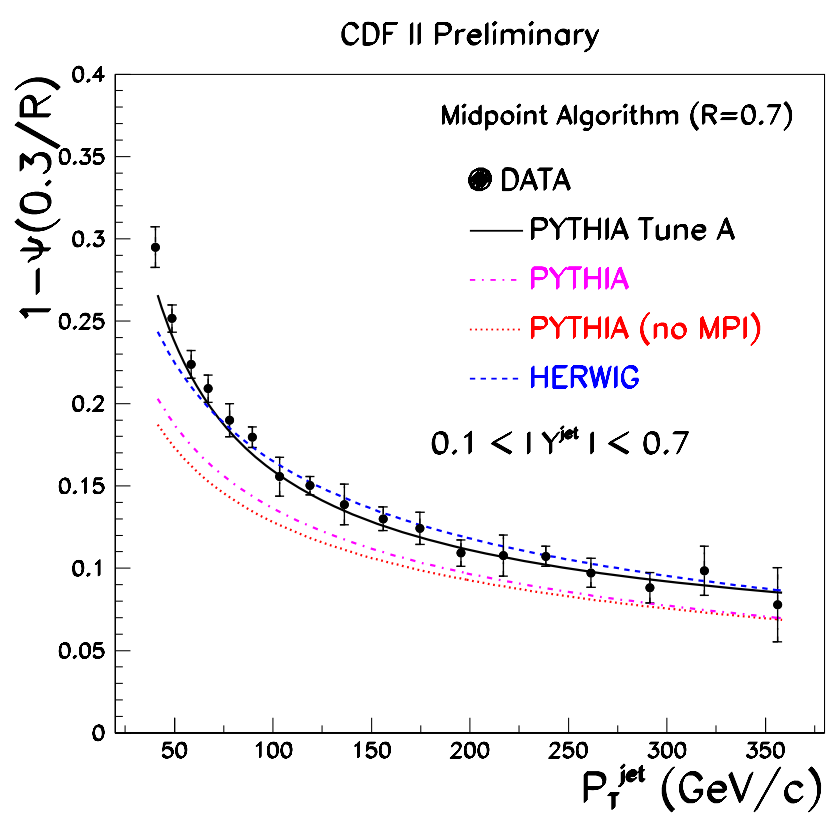

Fig. 3.3 The measured $1-\Psi(0.3 / R)$ as a function of $p_{\mathrm{T}}^{\text {jet }}$ for jets with $0.1<\left|y^{\text {jet }}\right|<0.7$ and $37 \mathrm{GeV} / c<p_{\mathrm{T}}^{\text {jet }}<380 \mathrm{GeV} / c$. Error bars indicate the statistical and systematic uncertainties added in quadrature. The predictions of PYTHIA-TUNE A (solid line), PYTHIA (dashed-dotted line), PYTHIA-(no MPI) (dotted line) and HERWIG (dashed line) are shown for comparison

HERWIG predictions describe the measured jet shapes well for $p_{\mathrm{T}}^{\text {jet }}>55 \mathrm{GeV}$ but produces jets that are too narrow at lower $p_{\mathrm{T}}^{\text {jet }}$.

\section{Dijet azimuthal decorrelations}

The D0 experiment has employed the dijet sample to study azimuthal decorrelations, $\Delta \phi_{\text {dijet }}$, between the two leading jets [21]. The normalized cross section,

$$
\frac{1}{\sigma_{\text {dijet }}} \frac{d \sigma}{d \Delta \phi_{\text {dijet }}},
$$

is sensitive to the spectrum of the gluon radiation in the event. The measurements has been performed in different regions of the leading jet $p_{\mathrm{T}}^{\text {jet }}$ starting at $p_{\mathrm{T}}^{\text {jet }}>75 \mathrm{GeV}$, where the second jet is required to have at least $p_{\mathrm{T}}^{\text {jet }}>40 \mathrm{GeV}$. Figure 4.1 shows the measured cross section compared to LO and NLO pQCD predictions [22]. The LO (non trivial) predictions for this observable, with at most three partons in the final state, is limited to $\Delta \phi_{\text {dijet }}>2 \pi / 3$, for which the three partons define a Mercedes-star topology. It presents a prominent peak at $\Delta \phi_{\text {dijet }}=\pi$ corresponding to the soft limit, for which the third parton is collinear to the direction of the two leading partons. The NLO predictions for this observable, with four partons in the final state, describes the measured $\Delta \phi_{\text {dijet }}$ distribution better except in the very

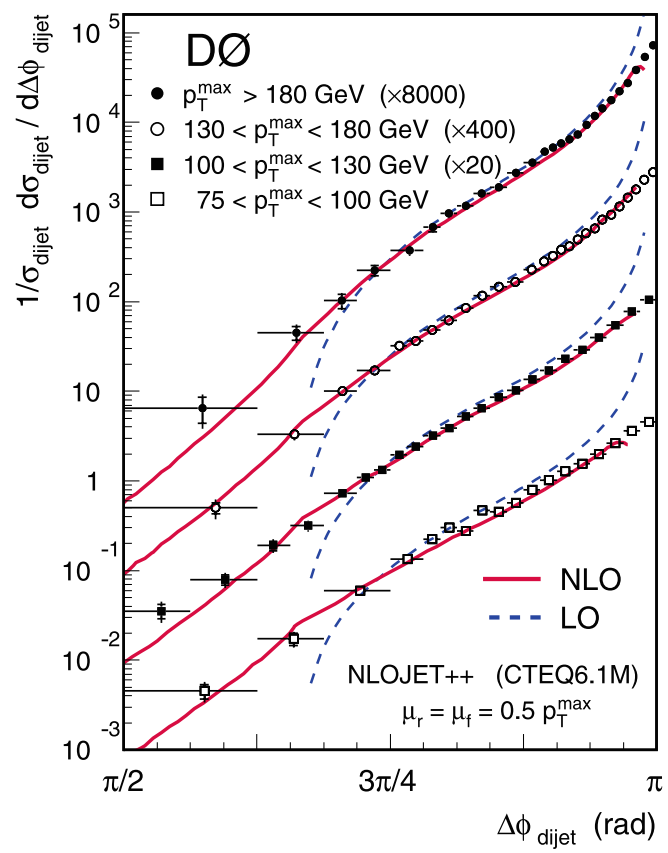

Fig. 4.1 Measured azimuthal decorrelations in dijet production for central jets compared to $\mathrm{pQCD}$ predictions in different regions of $p_{\mathrm{T}}^{\mathrm{jet}}$ of the leading jet

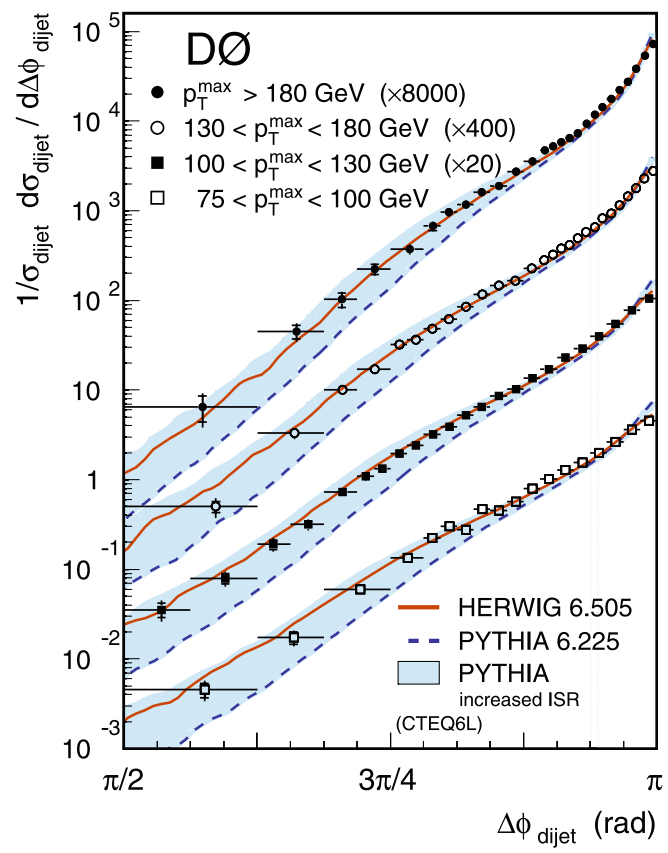

Fig. 4.2 Measured azimuthal decorrelations in dijet production for central jets compared to PYTHIA and HERWIG predictions in different regions of leading $p_{\mathrm{T}}^{\mathrm{jet}}$. The band covers PYTHIA predictions with different amount of initial-state soft-gluon radiation

high and very low regions of $\Delta \phi_{\text {dijet }}$, where additional soft contributions, corresponding to a resummed calculation, are necessary. A reasonable approximation to such calculations is provided by parton shower Monte Carlo programs. Fig- 
ure 4.2 presents the measured cross sections compared to PYTHIA and HERWIG predictions in different regions of $p_{\mathrm{T}}^{\mathrm{jet}}$. The PYTHIA samples with default parameters underestimate the gluon radiation at large angles. Different tunes of PYTHIA predictions are possible, which include an enhanced contribution from initial-state soft gluon radiation, to properly describe the azimuthal distribution. HERWIG also describes the data although tends to produce less radiation than PYTHIA close to the direction of the leading jets. This measurement clearly shows that angular correlations between jets can be employed to tune Monte Carlo predictions of soft gluon radiation in the final state.

\section{Prompt $\gamma$ and $\gamma+$ jet production}

The measurement of the inclusive photon production as a function of photon transverse momentum $p_{\mathrm{T}}^{\gamma}$ at the Tevatron constitutes a precise test of $\mathrm{pQCD}$ predictions since the energy scale of the electromagnetic calorimeters is well understood by the experiments. The measured cross section is partially dominated by contributions from quark-gluon scattering and therefore provides a powerful constrain of the gluon PDF at high- $x$. However, it is a rather difficult measurement where a good understanding of QCD backgrounds from $\pi^{0}$ and $\eta$ decays into photons is necessary. The D0 collaboration has presented results [23] on inclusive photon production, based on $326 \mathrm{pb}^{-1}$ of Run II data, in the region $\left|\eta^{\gamma}\right|<0.9$ and $23<p_{\mathrm{T}}^{\gamma}<300 \mathrm{GeV} / c$ (see Fig. 5.1). The measurements are compared to NLO pQCD predictions [24, 25]. Figure 5.2 shows the ratio data/NLO as a function of $p_{\mathrm{T}}^{\gamma}$. The measured cross section is well described by the theoretical prediction, where the latter presents uncertainties at the level of about $10 \%$. Future measurements based on few $\mathrm{fb}^{-1}$ of data will provide valuable information about the proton structure as well as impose strong constrains on the presence of new physics with very energetic photons in the final state.

The D0 Collaboration has complemented the inclusive measurements with detailed studies on direct photon production in association with jets, for jets in the region $p_{\mathrm{T}}^{\text {jet }}>$ $15 \mathrm{GeV} / c$ and $\left|y^{\text {jet }}\right|<2.5$. As shown in Fig. 5.3, different photon-jet rapidity correlations have been explored. The measurements are compared to NLO pQCD predictions, which provide a reasonable description of the data, expect in the region $1.5<\left|y^{\text {jet }}\right|<2.5$ and $y^{\text {jet }} \cdot y^{\gamma}>0$ where the theoretical predictions clearly underestimate the data at low $p_{\mathrm{T}}^{\gamma}$, beyond the quoted theoretical uncertainties. Therefore, these measurement will be input to future theoretical developments on prompt photon production.

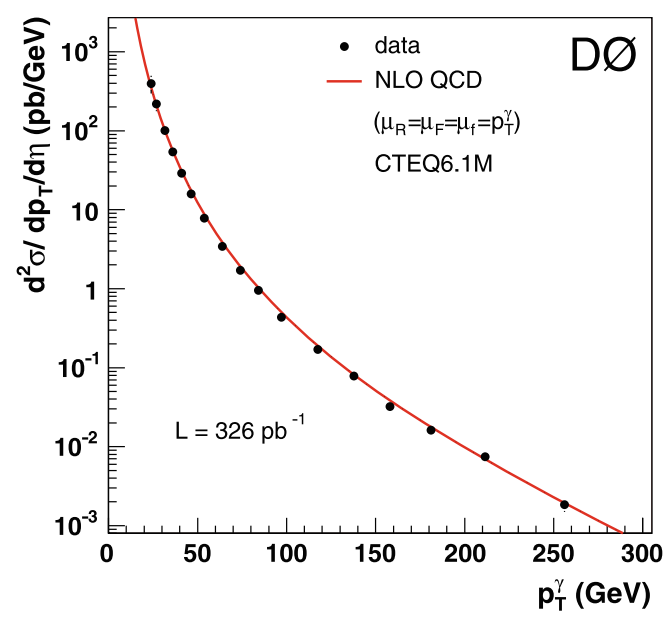

Fig. 5.1 Measured inclusive prompt photon cross section as a function of $p_{\mathrm{T}}^{\gamma}$ (dots) compared to $\mathrm{pQCD}$ NLO prediction (solid line)

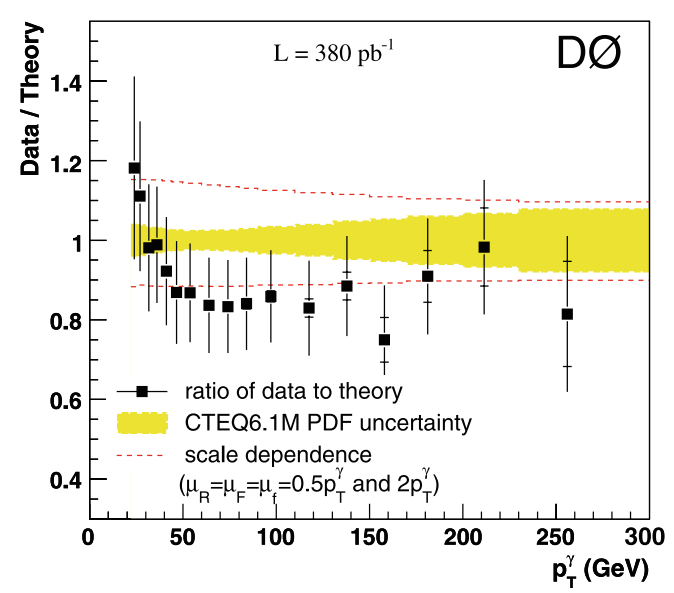

Fig. 5.2 Ratio data/theory as a function of $p_{\mathrm{T}}^{\gamma}$. The error bars show the uncertainty on the measurements. The band denotes the PDF uncertainty on the theoretical prediction and the dashed lines indicate the uncertainty from the variation of the renormalization/factorization scales in the PQCD NLO calculation

\section{Boson + jets production}

The study of the production of electroweak bosons in association with jets of hadrons in the final state constitutes a fundamental item in the high- $p_{\mathrm{T}}$ physics program at the Tevatron. These events are main backgrounds to many interesting physics processes like, for example, top production, the search for the SM Higgs, and supersymmetry. Therefore, during the last few years a significant effort is being made to develop and validate the necessary Monte Carlo tools to describe these complicated multijet final states. As a result, a number of leading-order Monte Carlo programs are available that describe boson + jet production processes up to large parton multiplicities [26-29], and NLO pQCD parton-level predictions are also available for a limited number of processes (up to boson +2 jets production) [30]. The 
Fig. 5.3 Ratio data/theory as a function of $p_{\mathrm{T}}^{\gamma}$ in $\gamma+$ jet events in different photon-jet rapidity configurations. The error bars show the uncertainty on the measurements. The band denotes the PDF uncertainty on the theoretical prediction (based on CTEQ6.5M PDFs) and the dotted lines indicate the uncertainty from the variation of the renormalization/ factorization scales in the $\mathrm{pQCD}$ NLO calculation. Ratios with theoretical predictions using different PDF sets are also shown

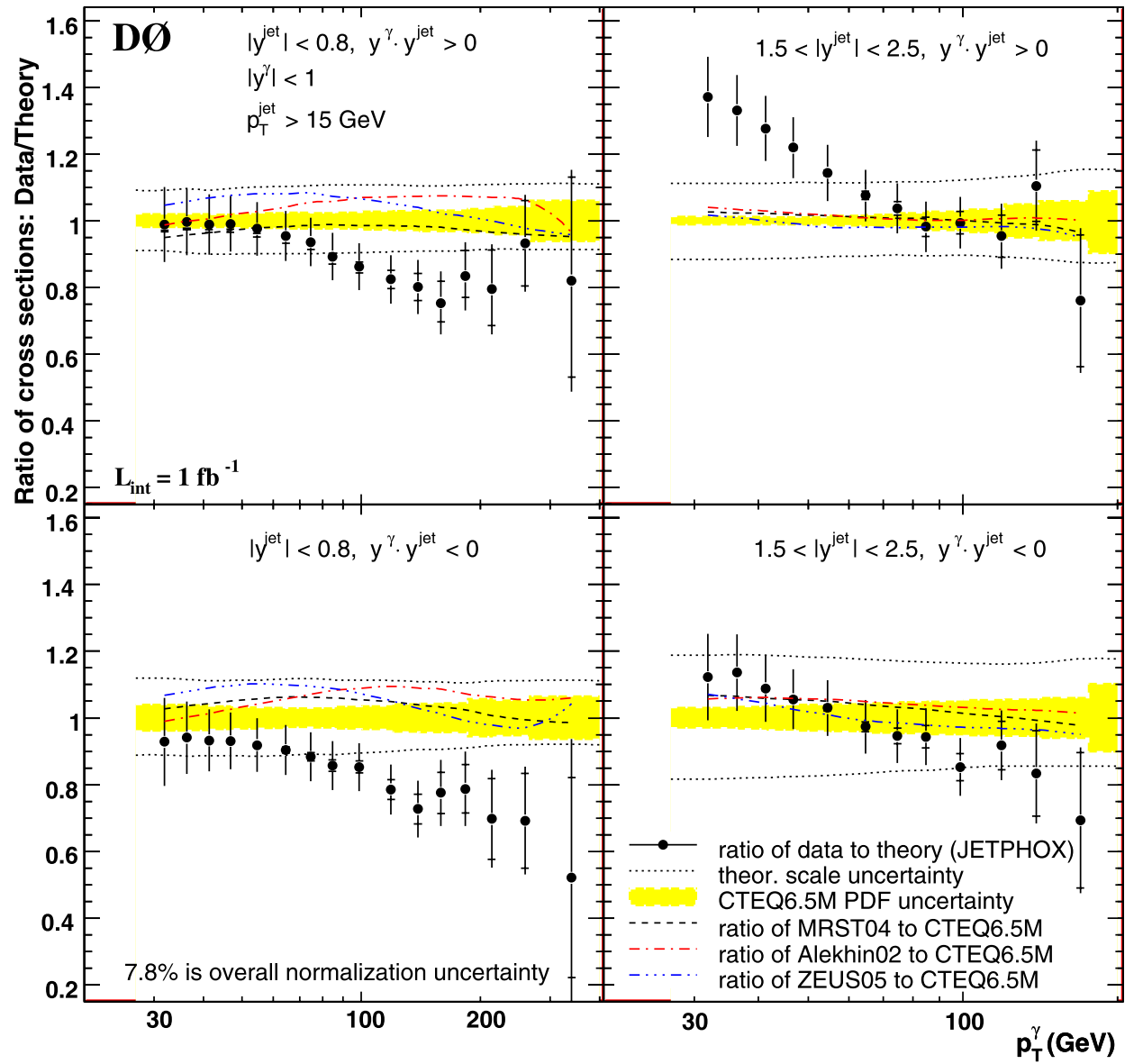

interface between parton level calculations and parton showers, necessary to describe the complexity of the observed hadronic final states, requires the introduction of different prescriptions to resolve resulting double counts in the gluon radiation across processes with different parton multiplicities. The theoretical prescriptions employed require validation using data. For this purpose, both CDF and D0 collaborations have performed a careful set of measurements on boson + jets production. Figure 6.1 shows the measured $p_{\mathrm{T}}^{\text {jet }}$ distribution for the $n$th jet in inclusive $W(\rightarrow e v)+\geq N_{\text {jet }}$ production by CDF [31], based on $320 \mathrm{pb}^{-1}$ of Run II data, where jets are reconstructed using a cone-based algorithm with radius $R=0.4$. This observable is particularly sensitive to the details on the implementation of the parton shower interface in the Monte Carlo models. The measurements are compared to leading-order Monte Carlo predictions as implemented in the ALPGEN program [27]. Figure 6.2 shows the ratio of data vs predictions for different Monte Carlo models, in addition to NLO pQCD parton-level predictions [30]. The observed shapes at low $p_{\mathrm{T}}^{\text {jet }}$ are attributed to the details of the underlying event implementation in the different models. Similarly, the D0 collaboration has carried out detail comparisons with different models in $\mathrm{Z} / \gamma^{*}+$ jets events [32] which indicate that SHERPA [29] pro-

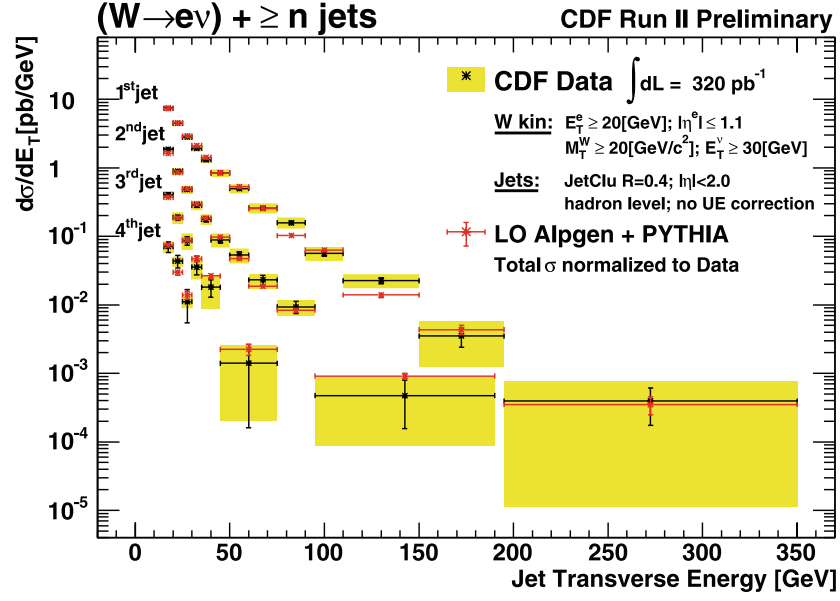

Fig. 6.1 Measured inclusive cross section as a function of $E_{\mathrm{T}}^{\text {jet }}$ for the $n$th jet in inclusive $W(\rightarrow e v)+\geq N_{\text {jet }}$ production. The measurements are compared to different leading-order Monte Carlo predictions normalized to the data

vides a reasonable description of the data at large jet multiplicities.

The CDF experiment has recently published precise measurements on jets in events with a $Z / \gamma^{*}$ boson in the final 
Fig. 6.2 Ratio of data vs predictions as a function of $E_{\mathrm{T}}^{\mathrm{jet}}$ for the $n$th jet in inclusive $W(\rightarrow e v)+\geq N_{\text {jet }}$ production, for different Monte Carlo models in addition to NLO pQCD predictions
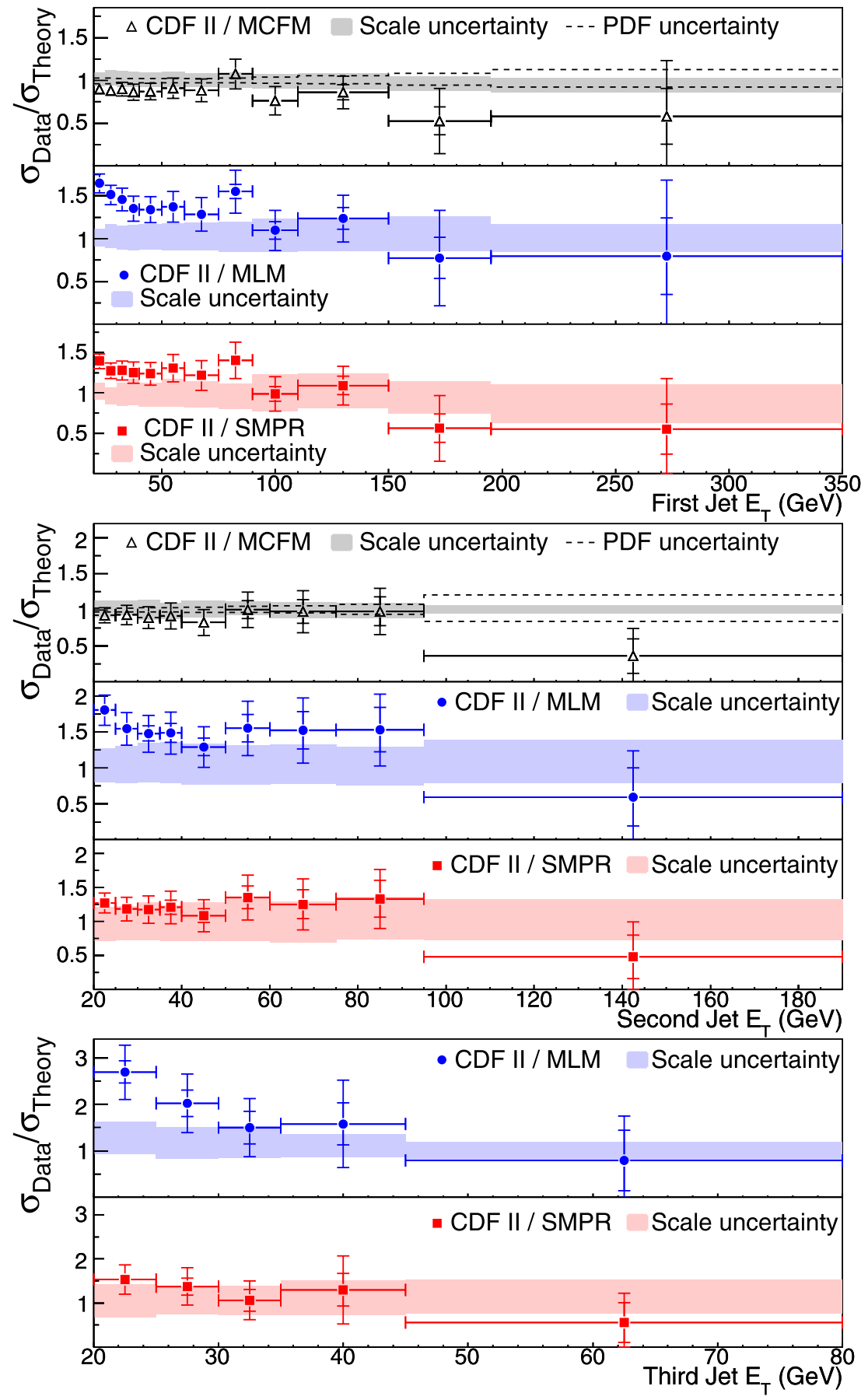

state [33]. Inclusive jet cross sections as a function of jet transverse momentum and jet multiplicity are measured for jets in the region $p_{\mathrm{T}}^{\text {jet }}>30 \mathrm{GeV} / c$ and $\left|y^{\text {jet }}\right|<2.3$, where the jets are reconstructed using a midpoint cone-based algorithm with radius $R=0.7$. The measurements are compared to NLO pQCD predictions [30] including non-perturbative contributions. Figure 6.3 shows the measured cross sections compared to MCFM predictions for the first two leading jets. In both cases, the measurements are well described by NLO
pQCD. Figure 6.4 shows the measured cross sections as a function of inclusive jet multiplicity compared to both LO and NLO pQCD predictions. The LO pQCD predictions underestimate the measured cross sections by a factor about 1.4 approximately independent of $N_{\text {jet }}$, while good agreement is observed between data and NLO pQCD predictions. The analysis of more data will make possible precise measurements at higher jet multiplicities for which NLO predictions are not available. 


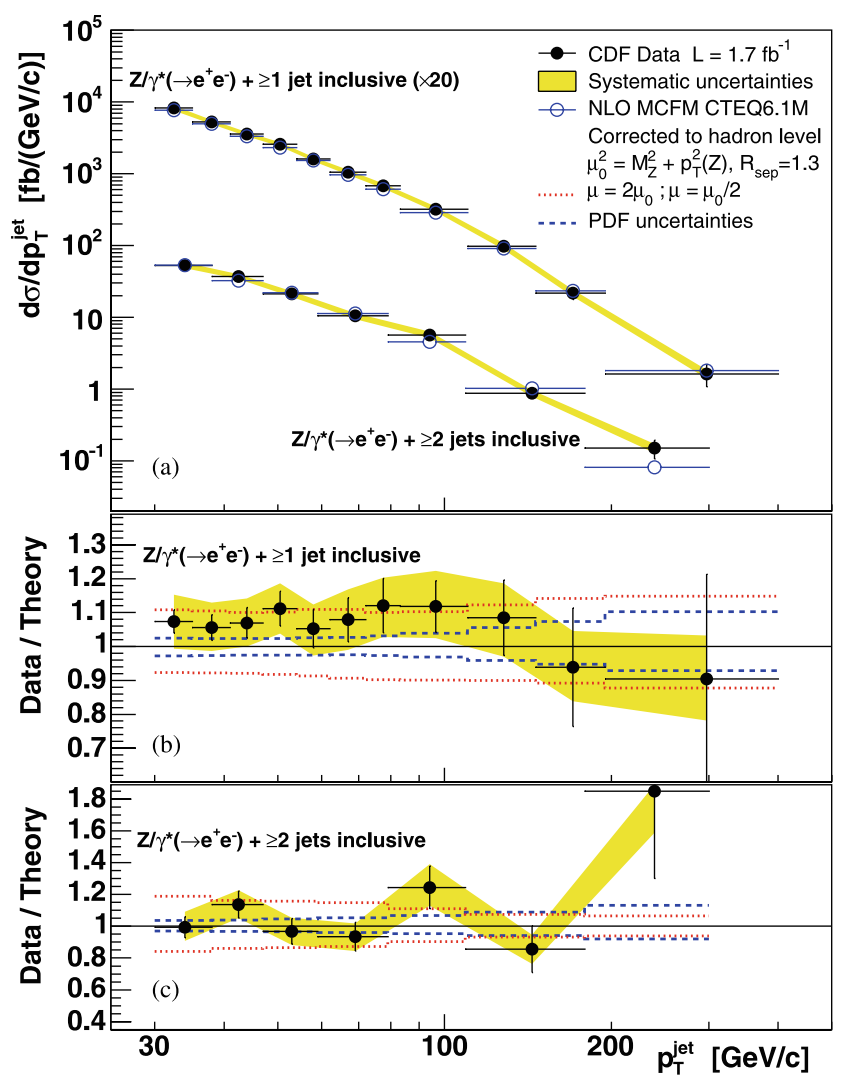

Fig. 6.3 (a) Measured inclusive jet differential cross section as a function of $p_{\mathrm{T}}^{\text {jet }}$ (black dots) in $Z / \gamma^{*}\left(\rightarrow e^{+} e^{-}\right)+$jets with $N_{\text {jet }} \geq 1,2$ compared to NLO pQCD predictions (open circles). The shaded bands show the total systematic uncertainty. (b and c) Data/theory ratio as a function of $p_{\mathrm{T}}^{\text {jet }}$ for $N_{\text {jet }} \geq 1$ and $N_{\text {jet }} \geq 2$, respectively. The dashed and dotted lines indicate the PDF uncertainty and the variation with the renomalization/factorization scale in the NLO pQCD predictions, respectively

\section{Notes on heavy-flavor jet production}

This contribution did not include a detailed discussion on inclusive $b$-jet production, or the production of electroweak bosons in association with heavy-flavor jets. However, great progress has been made also in these fronts. In the case of $b$-jet production, results indicate that good agreement is observed with NLO pQCD predictions for both inclusive onejet and dijet production [19]. In the $W / Z+$ jets final state, the situation is unfortunately less clear and will need further experimental and theoretical developments. While first measurements on $W+c$ production $[34,35]$ seem to be properly described by NLO pQCD predictions, significant discrepancies are reported between data and theoretical predictions in the case of $W / Z+b$ production [36]. This promises to be an important subject of discussion in the following years, given the fact that this final state is background for Higgs searches at the LHC.

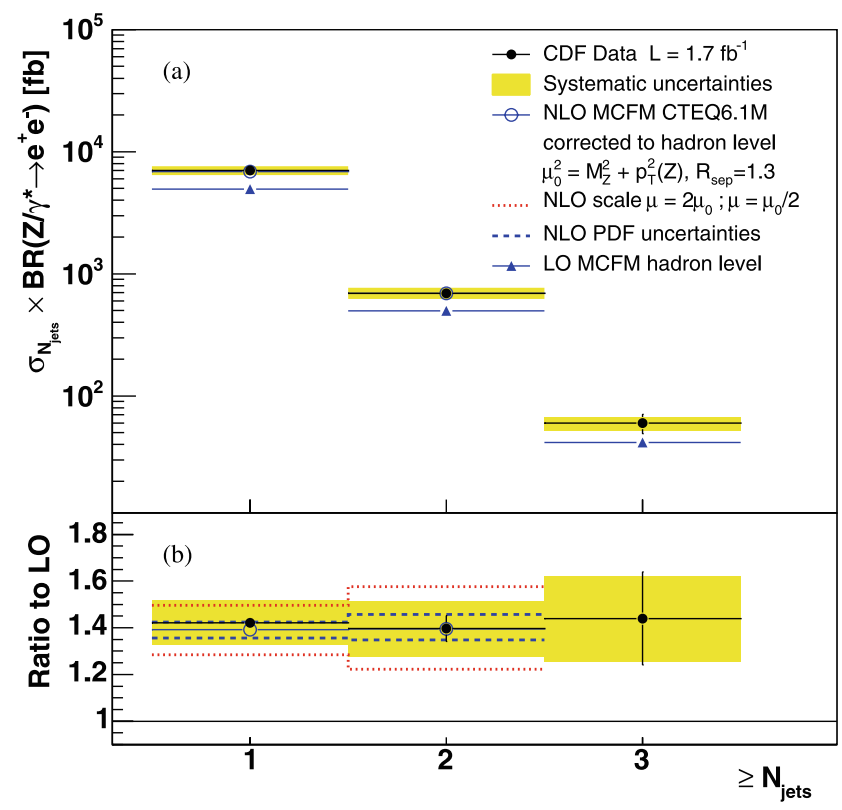

Fig. 6.4 (a) Measured cross section for inclusive jet production in $Z / \gamma^{*}\left(\rightarrow e^{+} e^{-}\right)$events as a function of $N_{\text {jet }}$ compared to LO and NLO pQCD predictions. The shaded bands show the total systematic uncertainty. (b) Ratio of data and NLO to LO pQCD predictions versus $N_{\text {jet }}$. The dashed and dotted lines indicate the PDF uncertainty and the variation with the renormalization/factorization scale in the NLO pQCD predictions, respectively

Acknowledgements I would like to thank the organizers for their kind invitation to the conference and the interesting discussions that they made possible.

\section{References}

1. B. Abbott et al. (DØ Collaboration), Phys. Rev. Lett. 82, 2451 (1999)

2. T. Affolder et al. (CDF Collaboration), Phys. Rev. D 64, 032001 (2001) [Erratum: Phys. Rev. D 65, 039903 (2002)]

3. A. Abulencia et al. (CDF Collaboration), Phys. Rev. Lett. 96, 122001 (2006)

4. A. Abulencia et al. (CDF Collaboration), Phys. Rev. D 75, 092006 (2007)

5. A. Abulencia et al. (CDF Collaboration), Phys. Rev. D 74, 071103(R) (2006)

6. T. Aaltonen et al. (CDF Collaboration), hep-ex/0807.2204

7. S. Catani et al., Nucl. Phys. B 406, 187 (1993)

8. S.D. Ellis, D.E. Soper, Phys. Rev. D 48, 3160 (1993)

9. G.C. Blazey et al., hep-ex/0005012

10. S.D. Ellis et al., hep-ph/0111434

11. W.T. Giele, E.W.N. Glover, D.A. Kosower, Nucl. Phys. B 403, 633 (1993)

12. V.M. Abazov et al. (DØCollaboration), Phys. Lett. B 525, 211 (2002)

13. V.M. Abazov et al. (DØCollaboration), Phys. Rev. Lett. 101, 062001 (2008)

14. T. Kluge, K. Rabbertz, M. Wobisch, hep-ph/0609285

15. Z. Nagy, Phys. Rev. D 68, 094002 (2003)

16. T. Sjöstrand et al., Comput. Phys. Commun. 135, 238 (2001)

17. G. Corcella et al., J. High Energy Phys. 0101, 010 (2001) 
18. T. Affolder et al. (CDF Collaboration), Phys. Rev. D 65, 092002 (2002)

19. URL: www-cdf.fnal.gov/physics/physics.html

20. D. Acosta et al. (CDF Collaboration), Phys. Rev. D 71, 112002 (2005)

21. V.M. Abazov et al. (DØCollaboration), Phys. Rev. Lett. 94, 221801 (2005)

22. Z. Nagy, Phys. Rev. Lett. 88, 122003 (2002)

23. V.M. Abazov et al. (DØCollaboration), Phys. Lett. B 639, 151 (2006) [Erratum June 2007]

24. T. Binoth et al., Eur. Phys. J. C 16, 311 (2000)

25. S. Catani et al., J. High Energy Phys. 05, 028 (2002)

26. F.A. Berends et al., Nucl. Phys. B 357, 32 (1991)

27. M.L. Mangano et al., J. High Energy Phys. 0307, 001 (2003)

28. F. Maltoni, T. Stelzer, J. High Energy Phys. 0302, 027 (2003)
29. T. Gleisburg et al., J. High Energy Phys. 0402, 056 (2004)

30. J. Campbell, R.K. Ellis, D. Rainwater, Phys. Rev. D 68, 094021 (2003)

31. T. Aaltonen et al. (CDF Collaboration), Phys. Rev. D 77, 011108(R) (2008)

32. www-d0.fnal.gov/Run2Physics/WWW/results/higgs.htm

33. T. Aaltonen et al. (CDF Collaboration), Phys. Rev. Lett. 100, $102001(2008)$

34. T. Aaltonen et al. (CDF Collaboration), Phys. Rev. Lett. 100, 091803 (2008)

35. V.M. Abazov et al. (DøCollaboration), Phys. Lett. B 666, 23 (2008)

36. A. Abulencia et al. (CDF Collaboration), Phys. Rev. D 74, 032008 (2006) 